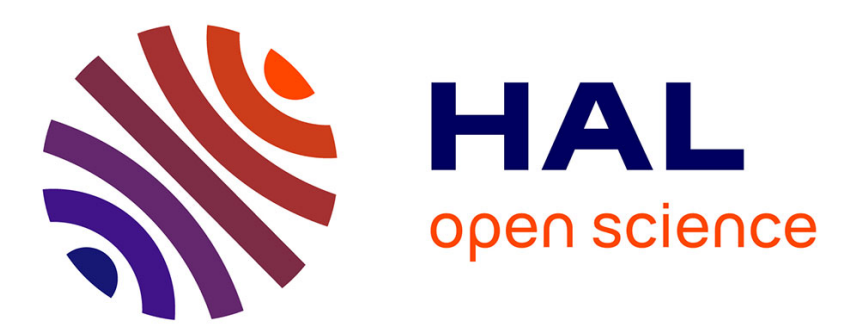

\title{
Well-positioned Closed Convex Sets and Well-positioned Closed Convex Functions
}

Samir Adly, Emil Ernst, Michel Théra

\section{To cite this version:}

Samir Adly, Emil Ernst, Michel Théra. Well-positioned Closed Convex Sets and Wellpositioned Closed Convex Functions. Journal of Global Optimization, 2004, 29 (4), pp.337-351. 10.1023/B:JOGO.0000047907.66385.5d . hal-00068258

\section{HAL Id: hal-00068258 \\ https://hal.science/hal-00068258}

Submitted on 18 Oct 2017

HAL is a multi-disciplinary open access archive for the deposit and dissemination of scientific research documents, whether they are published or not. The documents may come from teaching and research institutions in France or abroad, or from public or private research centers.
L'archive ouverte pluridisciplinaire $\mathbf{H A L}$, est destinée au dépôt et à la diffusion de documents scientifiques de niveau recherche, publiés ou non, émanant des établissements d'enseignement et de recherche français ou étrangers, des laboratoires publics ou privés. 


\title{
Well-positioned Closed Convex Sets and Well-positioned Closed Convex Functions
}

\author{
SAMIR ADLY ${ }^{1}$, EMIL ERNST $^{2}$ and MICHEL THÉRA ${ }^{1}$ \\ ${ }^{1}$ LACO, Université de Limoges, 123 Avenue A. Thomas, 87060 Limoges Cedex, France \\ (e-mail: $\{$ adly(adly)\}@thera@unilim.fr) \\ ${ }^{2}$ Laboratoire de Modelisation en Mécanique et Thermodynamique (LMMT), Casse 322, Faculté \\ de Sciences et Techniques de Saint Jérôme, Avenue Escadrille Normandie-Niemen 13397 \\ Marseille Cedex 20 (e-mail: emil.ernst@univ.u-3mrs.fr)
}

\begin{abstract}
We characterize the class of those closed convex sets which have a barrier cone with a nonempty interior. As a consequence, we describe the set of those proper extended-real-valued functionals for which the domain of their Fenchel conjugate has a nonempty interior. As an application, we study the stability of the solution set of a semi-coercive variational inequality.
\end{abstract}

Key words. barrier cone, convex analysis, Fenchel conjugate, recession analysis, semi-coercive functional, support functional, variational inequality.

\section{Introduction}

Closed convex sets for which the barrier cone has a nonempty interior, as well as proper extended-real-valued functionals for which the domain of their Fenchel conjugate is nonempty, are mathematical objects currently encountered in various areas of optimization theory and variational analysis (see for instance the class of well-behaved functions introduced by Auslender and Crouzeix in [5]).

This article provides, in the framework of general reflexive Banach spaces, geometric and analytical characterizations for this type of sets and functionals and extends in this way earlier partial results obtained in [3] in the context of separable Banach spaces.

Section 2 is dedicated to the study of the class of closed convex sets which have a barrier cone with a nonempty interior. Theorem 2.1 states that this class is identical to the class of closed convex sets having a geometrical property called well-positionedness. An analytical characterization of well-positioned sets - Proposition 2.1 - is equally obtained.

Similar results are deduced in Section 3 for functionals. A geometric characterization, valid for all the proper extended-real-valued functionals, and an analytical one, valid for convex lower semi-continuous functionals constitute the main results of the section. 
The article is completed by an application of Theorems 3.1 and 3.2 to the theory of variational inequalities. More precisely, the above mentioned results allow us to describe the class of all semi-coercive variational inequalities which have a nonempty solution set for any sufficiently small uniform perturbations of their data.

\section{Well-positioned Sets}

Unless otherwise stated we suppose that $X$ is a reflexive Banach space and we denote by $\langle\cdot, \cdot\rangle$ the duality pairing between $X$ and its continuous dual $X^{*}$, by $\|\cdot\|$ and $\|\cdot\|_{*}$ the norm and the dual norm on $X$ and $X^{*}$, respectively, and by $j: X^{*} \rightarrow X$ the duality mapping given by $\langle f, j(f)\rangle=\|f\|_{*}^{2},\|j(f)\|=\|f\|_{*}$, (see for example [8]). Due to a well-known renorming Theorem of Troyanski (see e.g. [7]) we can (and will) assume that the norms on $X$ and $X^{*}$ are locally uniformly rotund. This implies that the duality mapping $j$ is single-valued and norm-to-norm continuous. As standard, co A, $\overline{\text { co }} A, \overline{\text { span }} A$ are the convex, the closed convex hull and the closed linear span of the set $A \subset X$. Finally, we use the symbols $\mathbb{B}_{X}, \mathbb{B}_{X^{*}}$ and ' $\rightarrow$ ' for the open unit balls in $X$ and $X^{*}$ and the weak convergence, respectively. Following the usual terminology used in convex analysis (see Rockafellar [10] as a reference book), we recall that the recession cone of a closed convex $C$ is the closed convex cone $C_{\infty}$ defined by

$$
C_{\infty}=\left\{v \in X: \forall \lambda>0, x_{0} \in C, x_{0}+\lambda v \in C\right\} .
$$

If $\Phi: X \rightarrow \mathbb{R} \cup+\{\infty\}$ is an extended real-valued function, dom $\Phi$ is the set of all $x \in X$ for which $\Phi(x)$ is finite, and we say that $\Phi$ is proper if dom $\Phi \neq \emptyset$. When $\Phi$ is a proper lower semi-continuous convex function, the recession function $\Phi_{\infty}$ of $\Phi$ is the proper lower semi-continuous convex function whose epigraph is the recession cone of the epigraph of $\Phi$, i.e., epi $\Phi_{\infty}=(\text { epi } \Phi)_{\infty}$. Equivalently

$$
\Phi_{\infty}(x)=\lim _{t \rightarrow+\infty} \frac{\Phi\left(x_{0}+t x\right)}{t},
$$

where $x_{0}$ is any element such that $\Phi\left(x_{0}\right)$ is finite.

We denote by $\operatorname{Ker} \Phi_{\infty}=\left\{u \in X: \Phi_{\infty}(u)=0\right\}$, and we define the barrier cone of a set $C$ in $X$ as the set of all linear continuous functionals bounded from above on $C$, i.e.,

$$
\mathcal{B}(C)=\left\{g \in X^{*}: \sup _{x \in C}\langle g, x\rangle<+\infty\right\} .
$$

Finally, $C^{\circ}=\left\{g \in X^{*}:\langle g, x\rangle \leqslant 0 \quad \forall x \in X\right\}$ will denote the negative polar cone of the convex cone $C$ which is $C^{\perp}$ when $C$ is a closed subspace. As well-known, $\mathcal{B}(C)^{\circ}=C_{\infty}$ and by the Bipolar Theorem $\overline{\mathcal{B}(C)}=\left(C_{\infty}\right)^{\circ}$. A convex set $C$ is called linearly bounded if $C_{\infty}=\{0\}$.

DEFINITION 2.1. We say that a set $C \subset X$ is well-positioned, if there exists $x_{0} \in X$ and $g \in X^{*}$ such that:

$$
\left\langle g, x-x_{0}\right\rangle \geqslant\left\|x-x_{0}\right\|, \quad \forall x \in C .
$$


Equivalently, if we denote by $C_{g}:=\{x \in X:\langle g, x\rangle \geqslant\|x\|\}, C$ is well-positioned if and only if $C \subset x_{0}+C_{g}$ for some $x_{0} \in X$ and $g \in X^{*}$.

Remark 2.1. If a nonempty convex set $C$ is well-positioned, then $\operatorname{Int}\left(C_{\infty}\right)^{\circ}$ is nonempty. However, the converse fails to be true in infinite dimension.

Indeed, by definition we know that $C \subset u_{0}+C_{g}$ for some $u_{0} \in X$ and $g \in X^{*}$. Hence,

$$
C_{\infty} \subset\{u \in X:\langle g, u\rangle \geqslant\|u\|\}:=C_{g} .
$$

This implies that

$$
\left(C_{g}\right)^{\circ} \subset\left(C_{\infty}\right)^{\circ},
$$

and, as

$$
-g+\mathbb{B}_{X^{*}} \subset\left(C_{g}\right)^{\circ}
$$

we deduce that $\operatorname{Int}\left(C_{\infty}\right)^{\circ} \neq \emptyset$.

Finally, remark that every unbounded linearly bounded closed convex set provides a counterexample for the converse.

LEMMA 2.1. Let $C$ be a closed convex set containing no lines, and $y \in C$. For every $R>0$, let us define

$$
M_{y, R}^{C}=\left\{\frac{x-y}{\|x-y\|}: x \in C,\|x-y\| \geqslant R\right\} .
$$

The following two facts are equivalent:

(i) $C$ is well-positioned;

(ii) there is $R>0$ such that $0 \notin \overline{\mathrm{co}}\left(M_{y, R}^{C}\right)$.

Proof of Lemma 2.1: (i) $\Longrightarrow$ (ii).

Let $C$ be a well-positioned closed convex set, $\phi y \phi$ an element of $C$, and $\phi x_{0} \phi$ and $g$ two elements of $X$ and $X^{*}$ such that

$$
\left\langle g, x-x_{0}\right\rangle \geqslant\left\|x-x_{0}\right\|, \quad \forall x \in C .
$$

The first part of the proof consists of proving that if $R$ satisfies

$$
R>2\left(1+\|g\|_{*}\right)\left\|y-x_{0}\right\|,
$$

then 0 does not belong to $\overline{\mathrm{co}}\left(M_{y, R}^{C}\right)$.

Indeed, for every $x \in C$ such that $\|x-y\|>2\left(1+\|g\|_{*}\right)\left\|y-x_{0}\right\|$, relation (2.2) implies that

$$
\begin{aligned}
\left\langle g, \frac{x-y}{\|x-y\|}\right\rangle & \geqslant \frac{\left\|x-x_{0}\right\|}{\|x-y\|}-\left\langle g, \frac{y-x_{0}}{\|x-y\|}\right\rangle \\
& \geqslant 1-\frac{\left\|y-x_{0}\right\|}{\|x-y\|}-\|g\|_{*} \frac{\left\|y-x_{0}\right\|}{\|x-y\|} \\
& =1-\left(1+\|g\|_{*}\right) \frac{\left\|y-x_{0}\right\|}{\|x-y\|} \geqslant \frac{1}{2} .
\end{aligned}
$$


This yields,

$$
x \in \overline{\mathrm{co}}\left(M_{y, R}^{C}\right) \Longrightarrow\langle g, x\rangle \geqslant \frac{1}{2},
$$

and therefore $0 \notin \overline{\mathrm{co}}\left(M_{y, R}^{C}\right)$.

(ii) $\Longrightarrow$ (i) Now, let us consider a closed convex set $C$ and fix $y \in C$ such that $0 \notin \overline{\mathrm{co}}\left(M_{y, R}^{C}\right)$ for some $R>0$. We claim that if $z$ is the element of minimal norm in the set $\overline{\operatorname{co}} M_{y, R}^{C}$ and if $h=j^{-1}(z)$, then

$$
\left\langle\frac{3 h}{\|z\|^{2}}, x-\left(y-\frac{2 R}{\|z\|} z\right)\right\rangle \geqslant\left\|x-\left(y-\frac{2 R}{\|z\|} z\right)\right\|, \quad \forall x \in C .
$$

Indeed, for every $x \in C$ such that $\|x-y\| \geqslant R$, the vector $\frac{x-y}{\|x-y\|}$ belongs to $\overline{\operatorname{co}}\left(M_{y, R}^{C}\right)$, and by the definition of $z$ we have, $\left\langle h, \frac{x-y}{\|x-y\|}\right\rangle \geqslant\|z\|^{2}$. Accordingly, for each $x \in C$ such that $\|x-y\| \geqslant R$, we have

$$
\begin{aligned}
\left\langle\frac{3 h}{\|z\|^{2}}, x-\left(y-\frac{2 R}{\|z\|} z\right)\right\rangle & \geqslant 3\|x-y\|+3 \frac{2 R}{\|z\|} \\
& \geqslant 3\left(\|x-y\|+\frac{2 R}{\|z\|}\|z\|\right) \\
& \geqslant\left\|x-\left(y-\frac{2 R}{\|z\|} z\right)\right\| .
\end{aligned}
$$

If $\|x-y\| \leqslant R$, then

$$
\left\langle\frac{3 h}{\|z\|^{2}}, x-y\right\rangle \geqslant-\frac{3}{\|z\|^{2}}\|h\|_{*}\|x-y\| \geqslant-\frac{3 R}{\|z\|} .
$$

Hence,

$$
\begin{aligned}
\left\langle\frac{3 h}{\|z\|^{2}}, x-\left(y-\frac{2 R}{\|z\|} z\right)\right\rangle & \geqslant-\frac{3 R}{\|z\|}+\frac{6 R}{\|z\|}=\frac{3 R}{\|z\|} \\
& \geqslant 3 R=R+2 R \geqslant\|x-y\|+\frac{2 R}{\|z\|}\|z\| \\
& \geqslant\left\|x-\left(y-\frac{2 R}{\|z\|} z\right)\right\| \forall x \in y+R \mathbb{B}_{X} .
\end{aligned}
$$

The two previous relations prove (2.3). Setting $g=\frac{3 h}{\|z\|^{2}}$ and $x_{0}=y-\frac{2 R}{\|z\|} z$ in (2.3) we obtain (2.1), completing the proof of Lemma 2.1 .

The following result provides an analytical definition of well-positioned sets.

PROPOSITION 2.1. A nonempty closed convex set $C$ of a reflexive Banach space $X$ is well-positioned if and only if the following two assumptions are satisfied:

(a) C contains no lines;

(b) $\exists\left\{x_{n}\right\}_{n \in \mathbb{N}} \subset C,\left\|x_{n}\right\| \rightarrow+\infty$, such that $\frac{x_{n}}{\left\|x_{n}\right\|} \rightarrow 0$. 
Remark 2.2. Proposition 2.1 subsumes the fact that when $X$ is finite dimensional, a nonempty closed convex set is well-positioned if and only if $C_{\infty}$ is pointed, i.e., $C_{\infty} \cap-C_{\infty}=\{0\}$. In particular, every compact and convex set is well-positioned in $\mathbb{R}^{n}$.

Proof of Proposition 2.1. Let us first prove that every well-positioned convex closed set $C$ satisfies assumptions (a) and (b). As $C$ is well-positioned, there are $x_{0}$ in $X$ and $g$ in $X^{*}$ such that $C \subset x_{0}+C_{g}$. By the way of obtaining a contradiction, suppose that $C$ contains at least a line, that is, there are $x_{1}$ and $v$ in $X,\|v\|=1$, such that

$$
x_{1}+\lambda v \in C, \quad \forall \lambda \in \mathbb{R} .
$$

Accordingly,

$$
\begin{aligned}
\lambda\langle g, v\rangle+\left\langle g, x_{1}-x_{0}\right\rangle & =\left\langle g, x_{1}+\lambda v-x_{0}\right\rangle \geqslant\left\|x_{1}+\lambda v-x_{0}\right\| \\
& \geqslant|\lambda|-\left\|x_{1}-x_{0}\right\|, \quad \forall \lambda \in \mathbb{R} .
\end{aligned}
$$

For $\lambda=n$ and $\lambda=-n$, we obtain respectively

$$
\begin{gathered}
n\langle g, v\rangle+\left\langle g, x_{1}-x_{0}\right\rangle \geqslant n-\left\|x_{1}-x_{0}\right\| \text { and } \\
-n\langle g, v\rangle+\left\langle g, x_{1}-x_{0}\right\rangle \geqslant n-\left\|x_{1}-x_{0}\right\| .
\end{gathered}
$$

Summing up the two above relations, we deduce that

$$
\left\langle g, x_{1}-x_{0}\right\rangle+\left\|x_{1}-x_{0}\right\| \geqslant n \quad \forall n \in \mathbb{N}^{*},
$$

a contradiction establishing assumption (a).

Now, let us suppose that the assumption (b) fails, i.e., there exists a sequence $\left(x_{n}\right)_{n \in \mathbb{N}^{*}}$ such that $\left\|x_{n}\right\| \rightarrow \infty$ and $x_{n} /\left\|x_{n}\right\| \rightarrow 0$. Since $C$ is well-positioned, we obtain

$$
0=\lim _{n \rightarrow \infty}\left\langle g, x_{n} /\left\|x_{n}\right\|\right\rangle=\lim _{n \rightarrow \infty}\left\langle g, \frac{x_{n}-x_{0}}{\left\|x_{n}\right\|}\right\rangle \geqslant \lim _{n \rightarrow \infty} \frac{\left\|x_{n}-x_{0}\right\|}{\left\|x_{n}\right\|}=1,
$$

a contradiction. Hence, assumption (b) is satisfied, establishing the fact that every well-positioned closed convex set fulfills assumptions (a) and (b).

Conversely, let us consider a closed convex set $C$ satisfying relations (a) and (b), and suppose that $C$ is not well-positioned. Pick $y \in C$; Lemma 2.1 implies that $0 \in \overline{\mathrm{co}}\left(M_{y, n}^{C}\right)$ for every $n \in \mathbb{N}^{*}$. For every integer $n$, select $U_{n}$, a finite or countable subset of $M_{y, n}^{C}$ such that $0 \in \overline{\mathrm{co}}\left(U_{n}\right)$, and thus $R_{n} \subset C \backslash\left(y+n \mathbb{B}_{X}\right)$ where $R_{n}$ is such that $U_{n}=\left\{\frac{x}{\|x\|}: x \in R_{n}\right\}$. Let $X_{1}=\overline{\operatorname{span}}\left(y, \cup_{n \in \mathbb{N}^{*}} R_{n}\right)$ and $K=C \cap X_{1}$. The definitions of $X_{1}$ and $K$ imply that, for every $n \in \mathbb{N}^{*}, U_{n} \subseteq M_{y, n}^{K}$, and therefore

$$
0 \in \overline{\mathrm{co}}\left(U_{n}\right) \subseteq \overline{\mathrm{co}}\left(M_{y, n}^{K}\right)
$$


As $M_{y, R_{1}}^{K} \subseteq M_{y, R_{2}}^{K}$ whenever $R_{1} \geqslant R_{2}$, the previous relation yields that $0 \in \overline{\operatorname{co}}\left(M_{y, R}^{K}\right)$ for every $R>0$; from Lemma 2.1 it follows that the closed convex set $K=C \cap X_{1}$ is not well-positioned. As $X_{1}$ is separable, and $K$ contains no lines (being a subset of $C$ which, by virtue of assumption (a), contains no lines), a well-known result of Klee ([9]) implies that there is $f \in \mathcal{B}(K)$ such that

$$
\langle f, w\rangle<0 \quad \forall w \in K_{\infty}, w \neq 0 .
$$

Let us prove the following technical result.

LEMMA 2.2. Let $C$ be a closed convex set of a reflexive Banach space X. Suppose that for some $g \in X^{*} \backslash\{0\}$ and $t \in \mathbb{R}$ such that $t<\sup _{x \in C}\langle g, x\rangle$, the set $C_{g, t}=$ $\{x \in C:\langle g, x\rangle \geqslant t\}$ is bounded. Then, the set $C$ is well-positioned.

Proof of Lemma 2.2. Let $\bar{x}$ be an element of $C$ such that $t<\langle g, \bar{x}\rangle$. Since $C_{g, t}$ is bounded, there is $r>0$ such that $C_{g, t} \subset r \mathbb{B}_{X}$, and thus $C_{g, t} \subset\left(\bar{x}+2 r \mathbb{B}_{X}\right)$. Accordingly, for every $x \in C \backslash\left(\bar{x}+2 r \mathbb{B}_{X}\right)$, we have $\langle g, x\rangle<t$ and consequently,

$$
0<\frac{t-\langle g, x\rangle}{\langle g, \bar{x}-x\rangle}<1 .
$$

Thus,

$$
z(x)=\frac{t-\langle g, x\rangle}{\langle g, \bar{x}-x\rangle} \bar{x}+\frac{\langle g, \bar{x}\rangle-t}{\langle g, \bar{x}-x\rangle} x
$$

is a convex combination of $x$ and $\bar{x}$ and, accordingly, an element of $C$. Moreover, $\langle g, z(x)\rangle=t$ and therefore $z(x)$ necessarily belongs to $C_{g, t}$. Consequently,

$$
0<\|\bar{x}-z(x)\| \leqslant 2 r,
$$

that is

$$
\frac{\langle g, \bar{x}\rangle-t}{\langle g, \bar{x}-x\rangle}\|\bar{x}-x\| \leqslant 2 r, \quad \forall x \in C \backslash\left(\bar{x}+2 r \mathbb{B}_{X}\right) .
$$

Relation (2.5) implies that

$$
\left\langle g, \frac{x-\bar{x}}{\|x-\bar{x}\|}\right\rangle \leqslant \frac{t-\langle g, \bar{x}\rangle}{2 r}<0 \quad \forall x \in C,\|x-\bar{x}\| \geqslant 2 r .
$$

Consequently, $0 \notin \overline{\mathrm{co}}\left(M_{\bar{x}, 2 r}^{C}\right)$, and therefore (see Lemma 2.1) $C$ is well-positioned. The proof of Lemma 2.2 is thereby completed.

As $K$ is not well-positioned, from Lemma 2.2 it follows that the set

$$
K_{f, t}=\{x \in K: t \leqslant\langle f, x\rangle\}
$$


is unbounded for every $t<\sup _{x \in K}\langle f, x\rangle$. Accordingly, there is a sequence $\left(\zeta_{n}\right)_{n \in \mathbb{N}^{*}} \subset K_{f, t}$ such that $\left\|\zeta_{n}\right\| \rightarrow \infty$. Let $w$ be a weak cluster point of the bounded sequence $\left(\frac{\zeta_{n}}{\left\|\zeta_{n}\right\|}\right)_{n \in \mathbb{N}^{*}}$. As $\left\|\zeta_{n}\right\| \rightarrow \infty$, we have $w \in\left(K_{f, t}\right)_{\infty}$, and $w \neq 0$ by virtue of assumption (b). For every $s>0, y+s w \in K_{f, t}$, so

$$
t \leqslant\langle f, y+s w\rangle \leqslant \sup _{x \in K}\langle f, x\rangle \quad \forall s>0,
$$

that is

$$
\frac{t-\langle f, y\rangle}{s} \leqslant\langle f, w\rangle \leqslant \frac{\sup _{x \in K}\langle f, x\rangle-\langle f, y\rangle}{s} \quad \forall s>0
$$

relation which implies that $\langle f, w\rangle=0$. The contradiction between the previous equality and relation (2.4) completes the proof of Proposition 2.1.

We are now in position to state the main property of the well-positioned sets.

THEOREM 2.1. Let $C$ be a nonempty subset of a reflexive Banach space $X$. The following two conditions are equivalent:

(1) The barrier cone of $C$ has a nonempty interior;

(2) $C$ is well-positioned.

Moreover, if $\operatorname{Int} \mathcal{B}(C) \neq \emptyset$, then

$$
\operatorname{Int} \mathcal{B}(C)=\operatorname{Int}\left(C_{\infty}\right)^{\circ}
$$

Proof of Theorem 2.1. (2) $\Longrightarrow(1)$ : we prove that $\emptyset \neq \operatorname{Int}\left(C_{\infty}\right)^{\circ} \subseteq \mathcal{B}(C)$.

By Remark 2.1, pick $g \in \operatorname{Int}\left(C_{\infty}\right)^{\circ}$. In order to prove that $g \in \mathcal{B}(C)$, we first need to establish a technical result.

LEMMA 2.3. Suppose $C$ is well-positioned. Then, for every $g \in \operatorname{Int}\left(C_{\infty}\right)^{\circ}$, there are $R_{g}, \gamma_{g}>0$ such that

$$
\langle g, x\rangle \leqslant R_{g}-\gamma_{g}\|x\|, \quad \forall x \in C .
$$

Proof of Lemma 2.3. Let us denote by $C_{f}:=\{x \in X \mid\langle f, x\rangle \geqslant\|x\|\}$. Let $x_{0}$ and $f$ such that $C \subset x_{0}+C_{f}$, and take $\gamma_{g}$ such that $g+2\|f\|_{*} \gamma_{g} \mathbb{B}_{X^{*}} \subset\left(C_{\infty}\right)^{\circ}\left(\gamma_{g}\right.$ exists since $\left.g \in \operatorname{Int}\left(C_{\infty}\right)^{\circ}\right)$. For the purpose of obtaining a contradiction, suppose that there exists a sequence $\left(x_{n}\right)_{n \in \mathbb{N}^{*}}$ in $C$ such that

$$
\left\langle g, x_{n}\right\rangle \geqslant n-\gamma_{g}\left\|x_{n}\right\| .
$$

Noticing that

$$
\left(\|g\|_{*}+\gamma_{g}\right)\left\|x_{n}\right\| \geqslant\left\langle g, x_{n}\right\rangle+\gamma_{g}\left\|x_{n}\right\| \geqslant n,
$$


we deduce that $\lim _{n \rightarrow \infty}\left\|x_{n}\right\|=+\infty$. We may therefore define $t_{n}:=\frac{1}{\left\|x_{n}\right\|}$. Let $w$ be a weak cluster point of the sequence $\left(t_{n} x_{n}\right)_{n \in \mathbb{N}}$. Multiplying relation (2.8) by $t_{n}$ and passing to the limit as $n \rightarrow+\infty$, we obtain

$$
\langle g, w\rangle \geqslant \liminf _{n \rightarrow \infty} n t_{n}-\gamma_{g} \geqslant-\gamma_{g} .
$$

Since $g+2\|f\|_{*} \gamma_{g} \mathbb{B}_{X^{*}} \subset\left(C_{\infty}\right)^{\circ}$ and $w \in C_{\infty}$, we derive

$$
\langle g+h, w\rangle \leqslant 0, \text { for all } h \in X^{*},\|h\|_{*} \leqslant 2\|f\|_{*} \gamma_{g} .
$$

This yields

$$
\langle g, w\rangle \leqslant-2\|f\|_{*} \gamma_{g}\|w\| .
$$

We combine (2.9) and (2.10) to obtain

$$
-\gamma_{g} \leqslant\langle g, w\rangle \leqslant-2\|f\|_{*} \gamma_{g}\|w\|,
$$

that is

$$
\frac{1}{2} \geqslant\|f\|_{*}\|w\|
$$

On the other hand, when multiplied with $t_{n}$, relation

$$
\left\langle f, x_{n}-x_{0}\right\rangle \geqslant\left\|x_{n}-x_{0}\right\| \geqslant\left\|x_{n}\right\|-\left\|x_{0}\right\|
$$

yields

$$
\left\langle f, t_{n} x_{n}-t_{n} x_{0}\right\rangle \geqslant 1-t_{n}\left\|x_{0}\right\| .
$$

Passing to the limit as $n \rightarrow+\infty$ we obtain

$$
\langle f, w\rangle \geqslant 1 \text {. }
$$

Combining relations (2.11) and (2.12) gives

$$
\frac{1}{2} \geqslant\|f\|_{*}\|w\| \geqslant\langle f, w\rangle \geqslant 1,
$$

a contradiction, and the proof of Lemma 2.3 is achieved.

Inequality (2.7) implies that for all $x \in C$ we have $\langle g, x\rangle \leqslant R_{g}$, which yields $g \in \mathcal{B}(C)$, and therefore

$$
\operatorname{Int}\left(C_{\infty}\right)^{\circ} \subset \mathcal{B}(C) \text {. }
$$

As a result, $\operatorname{Int} \mathcal{B}(C) \neq \varnothing$, and the first part of the proof is completed. 
Now, let us prove that $(1) \Rightarrow(2)$ : By contradiction, suppose that Int $\mathcal{B}(C) \neq \emptyset$ and $C$ fails to be well-positioned. Pick $g$ in Int $\mathcal{B}(C)$ and $t \in \mathbb{R}$ such that $t<\langle g, \bar{x}\rangle$ for some $\bar{x} \in C$. By Lemma 2.2, the set

$$
C_{g, t}=\{x \in C: t \leqslant\langle g, x\rangle\}
$$

is unbounded. Hence, by the Banach-Steinhaus Theorem, there exists $h \in X^{*}$ and $x_{n} \in C_{g, t}$ such that:

$$
\left\langle h, x_{n}\right\rangle \geqslant n, \quad \forall n \in \mathbb{N} .
$$

For every fixed $\varepsilon>0$, we have

$$
\left\langle g+\varepsilon h, x_{n}\right\rangle \geqslant t+\varepsilon n .
$$

Therefore,

$$
g+\varepsilon h \notin \mathcal{B}(C), \quad \forall \varepsilon>0 .
$$

Hence,

$$
g \notin \operatorname{Int} \mathcal{B}(C),
$$

and the proof of the equivalence between (1) and (2) of Theorem 2.1 is thereby completed. In order to prove equality (2.6), let us remark that $\mathcal{B}(C) \subseteq\left(C_{\infty}\right)^{\circ}$, so

$$
\operatorname{Int} \mathcal{B}(C) \subseteq \operatorname{Int}\left(C_{\infty}\right)^{\circ}
$$

for every set $C$. If $\operatorname{Int} \mathcal{B}(C) \neq \emptyset$, then $C$ is well-positioned, so relation (2.13) holds; relation (2.6) follows from relations (2.14) and (2.13).

Theorem 2.1 and Lemma 2.3 have the following immediate consequence.

COROLLARY 2.1. For every $f \in \operatorname{Int} \mathcal{B}(C)$, there are $R_{f}, \gamma_{f}>0$ such that

$$
\langle f, u\rangle \leqslant R_{f}-\gamma_{f}\|u\|, \quad \forall u \in C .
$$

\section{Well-positioned Functionals}

When endowed with the standard norm

$$
\|\cdot\|_{X \times \mathbb{R}}: X \times \mathbb{R} \rightarrow \mathbb{R}_{+},\|(x, \mu)\|_{X \times \mathbb{R}}:=\sqrt{\|x\|^{2}+\mu^{2}}, \quad \forall(x, \mu) \in X \times \mathbb{R},
$$

the linear space $X \times \mathbb{R}$ becomes a reflexive Banach space whose continuous dual is $X^{*} \times \mathbb{R}$ endowed with the standard norm:

$$
\|\cdot\|_{X^{*} \times \mathbb{R}}: X^{*} \times \mathbb{R} \rightarrow \mathbb{R}_{+},\|(f, \lambda)\|_{X^{*} \times \mathbb{R}}:=\sqrt{\|f\|_{*}^{2}+\lambda^{2}}, \quad \forall(f, \lambda) \in X^{*} \times \mathbb{R} ;
$$


the duality pairing is given by

$$
\langle(f, \lambda),(x, \mu)\rangle_{X^{*} \times \mathbb{R}, X \times \mathbb{R}}=\langle f, x\rangle+\lambda \mu, \quad \forall(f, \lambda) \in X^{*} \times \mathbb{R}, \quad(x, \mu) \in X \times \mathbb{R},
$$

and $J: X^{*} \times \mathbb{R} \rightarrow X \times \mathbb{R}$, given by

$$
J(f, \lambda)=(j(f), \lambda), \quad \forall(f, \lambda) \in X^{*} \times \mathbb{R},
$$

is the duality mapping between $X^{*} \times \mathbb{R}$ and $X \times \mathbb{R}$. Given an extended-real valued function $\Psi: X \rightarrow \mathbb{R} \cup\{+\infty\}$, recall that the Fenchel conjugate of $\Psi$ is the function $\Psi^{*}: X^{*} \rightarrow \mathbb{R} \cup\{+\infty\}$ given by

$$
\Psi^{*}(f):=\sup _{x \in X}\{\langle f, x\rangle-\Psi(x)\} .
$$

Obviously, the domain of $\Psi^{*}$ is connected to the barrier cone of the epigraph of $\Psi$ through the following equivalence

$$
g \in \operatorname{Int} \operatorname{dom} \Psi^{*} \Longleftrightarrow(g,-1) \in \mathcal{B}(\text { epi } \Psi) .
$$

This yields, dom $\Psi^{*} \times\{-1\}$ is the intersection of the barrier cone of epi $\Psi$ with the hyperplane $X^{*} \times\{-1\}$ of $X^{*} \times \mathbb{R}$ :

$$
\operatorname{dom} \Psi^{*} \times\{-1\}=\mathcal{B}(\text { epi } \Psi) \cap\left(X^{*} \times\{-1\}\right) .
$$

Standard convex analysis techniques allow us to prove that the domain of the Fenchel conjugate is nonempty if and only if the barrier cone of the epigraph has a nonempty interior.

DEFINITION 3.1. We say that a proper convex lower semicontinuous functional $\Psi: X \rightarrow \mathbb{R} \cup\{+\infty\}$ is well-positioned if the epigraph of $\Psi$,

$$
\text { epi } \Psi=\{(x, \lambda) \in X \times \mathbb{R}: \lambda \geqslant \Psi(x)\},
$$

is a well-positioned subset of $X \times \mathbb{R}$.

Theorem 2.1 may now be rephrased into the following geometrical characterization of functionals for which the domain of their Fenchel conjugate has a non void interior.

PROPOSITION 3.1. Let $\Psi$ be a proper lower semicontinuous convex function on a reflexive Banach space. Then,

$$
\text { Int } \operatorname{dom} \Psi^{*} \neq \emptyset \Longleftrightarrow \Psi \text { is well-positioned. }
$$

In the case of proper lower semi-continuous convex extended-real-valued functionals which are bounded from below, Proposition 2.1 provides an analytical characterization of the well-positionedness which is easier to use. 
THEOREM 3.1. A proper lower semi-continuous convex functional $\Psi$ defined on a reflexive Banach space $X$ is well-positioned if and only if the two following assumptions hold:

(a) $\operatorname{Ker}\left(\Psi_{\infty}\right)$ contains no lines;

(b) $\exists\left(x_{n}\right)_{n \in \mathbb{N}^{*}} C \operatorname{dom} \Psi,\left\|x_{n}\right\| \rightarrow+\infty$, such that $\frac{x_{n}}{\left\|x_{n}\right\|} \rightarrow 0$ and $\frac{\Psi\left(x_{n}\right)}{\left\|x_{n}\right\|} \rightarrow 0$.

Proof of Theorem 3.1. By virtue of Proposition 2.1, we have only to prove that statement (a) is equivalent to $\left(\mathrm{a}^{*}\right)$ epi $\Psi$ contains no lines, and that statement (b) is equivalent to $\left(\mathrm{b}^{*}\right) \nexists\left(x_{n}, \mu_{n}\right) \in \operatorname{epi} \Psi,\left\|\left(x_{n}, \mu_{n}\right)\right\|_{X \times \mathbb{R}} \rightarrow \infty$, such that $\frac{\left(x_{n}, \mu_{n}\right)}{\left\|\left(x_{n}, \mu_{n}\right)\right\|_{X \times \mathbb{R}}} \rightarrow 0$.

Given a closed convex set $C$, let $L(C)$ denote the maximal closed linear space contained in $C$, i.e.,

$$
L(C)=C_{\infty} \cap\left(-C_{\infty}\right)
$$

For $C=$ epi $\Psi$, we have

$$
L(\text { epi } \Psi)=(\operatorname{epi} \Psi)_{\infty} \cap\left(-(\operatorname{epi} \Psi)_{\infty}\right)=\operatorname{epi}\left(\Psi_{\infty}\right) \cap\left(-\operatorname{epi}\left(\Psi_{\infty}\right)\right) ;
$$

as $\Psi$ is bounded from below, $\Psi_{\infty} \geqslant 0$. Hence,

$$
\operatorname{epi}\left(\Psi_{\infty}\right) \cap\left(-\operatorname{epi}\left(\Psi_{\infty}\right)\right)=\operatorname{Ker}\left(\Psi_{\infty}\right) \times\{0\} \cap\left(-\operatorname{Ker}\left(\Psi_{\infty}\right) \times\{0\}\right) .
$$

Consequently,

$$
L(\operatorname{epi} \Psi)=L\left(\operatorname{Ker}\left(\Psi_{\infty}\right)\right) \times\{0\} .
$$

Hence, $L($ epi $\Psi)=\{0\}$ if and only if $L\left(\operatorname{Ker} \Psi_{\infty}\right)=\{0\}$. Thus (a) is equivalent to $\left(a^{*}\right)$. In order to prove the equivalence between $(b)$ and $\left(b^{*}\right)$, let us first consider a sequence $\left(x_{n}, \mu_{n}\right)_{n \in \mathbb{N}^{*}} \in$ epi $\Psi$ such that

$$
\left\|\left(x_{n}, \mu_{n}\right)\right\|_{X \times \mathbb{R}} \rightarrow \infty \text { and } \frac{\left(x_{n}, \mu_{n}\right)}{\left\|\left(x_{n}, \mu_{n}\right)\right\|_{X \times \mathbb{R}}} \rightarrow 0 .
$$

As

$$
\lim _{n \rightarrow \infty}\left\langle f, \frac{x_{n}}{\left\|\left(x_{n}, \mu_{n}\right)\right\|_{X \times \mathbb{R}}}\right\rangle=\lim _{n \rightarrow \infty}\left\langle(f, 0), \frac{\left(x_{n}, \mu_{n}\right)}{\left\|\left(x_{n}, \mu_{n}\right)\right\|_{X \times \mathbb{R}}}\right\rangle_{X^{*} \times \mathbb{R}, X \times \mathbb{R}}=0,
$$

for every $f \in X^{*}$, it follows that

$$
\frac{x_{n}}{\left\|\left(x_{n}, \mu_{n}\right)\right\|_{X \times \mathbb{R}}} \rightarrow 0 .
$$

On the other side,

$$
\lim _{n \rightarrow \infty} \frac{\mu_{n}}{\left\|\left(x_{n}, \mu_{n}\right)\right\|_{X \times \mathbb{R}}}=\lim _{n \rightarrow \infty}\left\langle(0,1), \frac{\left(x_{n}, \mu_{n}\right)}{\left\|\left(x_{n}, \mu_{n}\right)\right\|_{X \times \mathbb{R}}}\right\rangle_{X^{*} \times \mathbb{R}, X \times \mathbb{R}}=0 .
$$


Hence,

$$
\lim _{n \rightarrow \infty} \frac{\left\|x_{n}\right\|}{\left\|\left(x_{n}, \mu_{n}\right)\right\|_{X \times \mathbb{R}}}=1 .
$$

Since $\left\|\left(x_{n}, \mu_{n}\right)\right\|_{X \times \mathbb{R}} \rightarrow+\infty$, it follows from (3.19) that

$$
\lim _{n \rightarrow \infty}\left\|x_{n}\right\|=+\infty
$$

from (3.17) and (3.19) we obtain

$$
\frac{x_{n}}{\left\|x_{n}\right\|}=\frac{\left\|\left(x_{n}, \mu_{n}\right)\right\|_{X \times \mathbb{R}}}{\left\|x_{n}\right\|} \frac{x_{n}}{\left\|\left(x_{n}, \mu_{n}\right)\right\|_{X \times \mathbb{R}}} \rightarrow 1.0=0 .
$$

The functional $\Psi$ is bounded from below, so there is $k \in \mathbb{R}$ such that

$$
k \leqslant \Psi\left(x_{n}\right) \leqslant \mu_{n}
$$

Finally, from (3.18) and (3.21) we get

$$
0=\lim _{n \rightarrow \infty} \frac{k}{\left\|x_{n}\right\|} \leqslant \lim _{n \rightarrow \infty} \frac{\Psi\left(x_{n}\right)}{\left\|x_{n}\right\|} \leqslant \lim _{n \rightarrow \infty} \frac{\mu_{n}}{\left\|x_{n}\right\|}=0 .
$$

Relations (3.20), (3.21) and (3.22) prove that whenever the sequence $\left(\left(x_{n}, \mu_{n}\right)\right)_{n \in \mathbb{N}^{*}}$ fulfills assumption $\left(\mathrm{b}^{*}\right)$, the sequence $\left(x_{n}\right)_{n \in \mathbb{N}^{*}}$ satisfies (b); consequently, (b) implies ( $\left.\mathrm{b}^{*}\right)$. Let now $\left(x_{n}\right)_{n \in \mathbb{N}^{*}} \in \operatorname{dom} \Psi$ such that $\left\|x_{n}\right\| \rightarrow+\infty$, $\frac{x_{n}}{\left\|x_{n}\right\|} \rightarrow 0$ and $\frac{\Psi\left(x_{n}\right)}{\left\|x_{n}\right\|} \rightarrow 0$. As $\left\|\left(x_{n}, \Psi\left(x_{n}\right)\right)\right\|_{X \times \mathbb{R}} \geqslant\left\|x_{n}\right\|$, it follows that

$$
\lim _{n \rightarrow \infty}\left\|\left(x_{n}, \Psi\left(x_{n}\right)\right)\right\|_{X \times \mathbb{R}}=+\infty ;
$$

as $\frac{\Psi\left(x_{n}\right)}{\left\|x_{n}\right\|} \rightarrow 0$, we derive

$$
\lim _{n \rightarrow \infty} \frac{\left\|x_{n}\right\|}{\left\|\left(x_{n}, \Psi\left(x_{n}\right)\right)\right\|_{X \times \mathbb{R}}}=1 .
$$

Let $(f, \lambda) \in X^{*} \times \mathbb{R}$; from (3.23) and (3.24) it follows that

$$
\begin{aligned}
\lim _{n \rightarrow \infty}\left\langle(f, \lambda), \frac{\left(x_{n}, \Psi\left(x_{n}\right)\right)}{\left\|\left(x_{n}, \Psi\left(x_{n}\right)\right)\right\|_{X \times \mathbb{R}}}\right\rangle & \\
= & \lim _{n \rightarrow \infty}\left(\left\langle f, \frac{x_{n}}{\left\|x_{n}\right\|}\right\rangle \frac{\left\|x_{n}\right\|}{\left\|\left(x_{n}, \Psi\left(x_{n}\right)\right)\right\|_{X \times \mathbb{R}}}+\right. \\
& +\lambda \lim _{n \rightarrow \infty}\left(\frac{\Psi\left(x_{n}\right)}{\left\|x_{n}\right\|} \frac{\left\|x_{n}\right\|}{\left\|\left(x_{n}, \Psi\left(x_{n}\right)\right)\right\|_{X \times \mathbb{R}}}=0.1+\lambda \cdot 0 \cdot 1=0 .\right.
\end{aligned}
$$


Consequently,

$$
\frac{\left(x_{n}, \Psi\left(x_{n}\right)\right)}{\left\|\left(x_{n}, \Psi\left(x_{n}\right)\right)\right\|_{X \times \mathbb{R}}} \rightarrow 0 .
$$

Relations (3.23) and (3.25) show that the sequence $\left(\left(x_{n}, \Psi\left(x_{n}\right)\right)\right)_{n \in \mathbb{N}^{*}}$ fulfills $\left(b^{*}\right)$ provided that the sequence $\left(x_{n}\right)_{n \in \mathbb{N}^{*}}$ satisfies (b). Accordingly, ( $\left.\mathrm{b}^{*}\right)$ implies (b), and the proof of Theorem 3.1 is completed.

Let $f \in \operatorname{Int} \mathcal{B}(\Psi)$. By virtue of Corollary 2.1, we deduce the existence of two constants $R_{f}$ and $\gamma_{f}>0$ such that

$$
\langle(f,-1),(u, \lambda)\rangle_{\left(X^{*} \times \mathbb{R}, X \times \mathbb{R}\right)} \leqslant R_{f}-\gamma_{f}\|(u, \lambda)\|_{X \times \mathbb{R}}, \quad \forall u \in \operatorname{dom} \Psi, \lambda \geqslant \Psi(u) .
$$

As $\|(u, \lambda)\|_{X \times \mathbb{R}} \geqslant\|u\|$, by setting $\lambda=\Psi(u)$ in the previous inequality we derive

$$
\begin{aligned}
& f \in \operatorname{Int} \operatorname{dom} \Psi^{*} \Longrightarrow \exists \gamma_{f}, R_{f}>0 \text { s.t. }\langle f, u\rangle+\gamma_{f}\|u\|_{R_{f}} \leqslant R_{f}+\Psi(u), \\
& \quad \forall u \in X .
\end{aligned}
$$

We have thus proved the following result.

THEOREM 3.2. $g \in \operatorname{Int} \operatorname{dom} \Psi^{*}$ if and only if the functional $\Psi-g$ is coercive, i.e.,

$$
\liminf _{\|x\| \rightarrow+\infty} \frac{\Psi(x)-\langle g, x\rangle}{\|x\|}>0 .
$$

Remark 3.1. From the previous result it follows that 0 belongs to the interior of the domain of $\Psi^{*}$ (in other words this means that $\Psi$ is well-behaved) if and only if $\Psi$ is coercive.

\section{Stability of the Existence of the Solution for Semi-coercive Variational Inequalities}

In various problems in optimization and in variational analysis (see the case of well-behaved functionals, for instance), it is a natural question to ask under which conditions the interior of the domain of the Fenchel conjugate of a given functional is nonempty. One of the problem leading to such conditions concerns (see [3] and [4]) the stability of the solution set of a semi-coercive variational inequality

$V I(A, f, \Phi, K)$ : find $u \in K \cap \operatorname{dom} \Phi$ such that

$$
\langle A u-f, v-u\rangle+\Phi(v)-\Phi(u) \geqslant 0, \quad \forall v \in K,
$$


where $K$ is a closed convex set in a reflexive Banach space $X, f$ is a continuous linear functional on $X, \Phi: X \rightarrow \mathbb{R} \cup\{+\infty\}$ is a proper lower semi-continuous and convex functional that we assume to be bounded from below, $K \cap \operatorname{dom} \Phi \neq \emptyset$, where $\operatorname{dom} \Phi:=\{x \in X: \Phi(x)<+\infty\}$, and $A$ is a semi-coercive operator from $X$ to $X^{*}$, that is

$$
\begin{aligned}
& \langle A v-A u, v-u\rangle \geqslant \kappa\left(\operatorname{dist}_{U}(v-u)\right)^{2} \quad \forall u, v \in X \\
& A(x+u)=A(x) \quad \forall x \in X \text { and } u \in U, \text { and } A(X) \subseteq U^{\perp},
\end{aligned}
$$

for some positive constant $\kappa$ and some closed subspace $U$ of $X$. We suppose furthermore that $A$ is pseudomonotone in the sense of Brezis ([6], p. 142).

In other words, we characterize all data $(A, f, \Phi, K)$ for which there is some $\varepsilon>0$ such that the variational inequality $\operatorname{VI}\left(A_{\varepsilon}, f_{\varepsilon}, \Phi_{\varepsilon}, K_{\varepsilon}\right)$ has solutions for every instance involving a bounded and semi-coercive operator $A_{\varepsilon}$, a linear continuous functional $f_{\varepsilon}$, a proper lower semi-continuous and convex functional $\Phi_{\varepsilon}$ that is bounded from below, and a closed convex set $K_{\varepsilon}$ such that $K_{\varepsilon} \cap \operatorname{dom} \Phi \neq \emptyset$, and

$$
\begin{aligned}
& \left\|A(x)-A_{\varepsilon}(x)\right\|_{*}<\varepsilon, \quad \forall x \in X \\
& \left\|f-f_{\varepsilon}\right\|_{*}<\varepsilon, \\
& K \subset K_{\varepsilon}+\varepsilon \mathbb{B}_{X} \text { and } K_{\varepsilon} \subset K+\varepsilon \mathbb{B}_{X}, \\
& \Phi(x)-\varepsilon \leqslant \Phi_{\varepsilon}(x) \leqslant \Phi(x)+\varepsilon, \quad \forall x \in X .
\end{aligned}
$$

In this framework, it was proved ([3] Proposition 3.1) that a sufficient and necessary condition ensuring the uniform stability of the solution set of the given variational inequality is that $f$ belongs to the interior of the domain of the Fenchel conjugate of an energy-like functional:

Int $R(A, \Phi, K)=\operatorname{Int} \operatorname{dom} \Psi^{*}$,

where

$$
R(A, \Phi, K)=\left\{f \in X^{*} \mid V . I .(A, f, \Phi, K) \text { has at least a solution }\right\} .
$$

is the resolvent set, and

$$
\Psi(x):=\kappa\left(\operatorname{dist}_{U}(x)\right)^{2}+I_{K}(x)+\Phi(x) \quad \forall x \in X,
$$

$I_{K}$ denoting the indicator function of $K$, i.e., $I_{K}(x)=0$ if $x \in K$ and $+\infty$ else.

Theorem 3.1 may now be used to obtain the following analytical characterization of the stability of the solution set.

The variational inequality V.I. $\left(A_{\varepsilon}, f_{\varepsilon}, \Phi_{\varepsilon}, K_{\varepsilon}\right)$ has solutions for every sufficiently small uniform perturbations $A_{\varepsilon}, f_{\varepsilon}, \Phi_{\varepsilon}, K_{\varepsilon}$, if and only if the following three conditions hold:

(i) The set $U \cap K_{\infty} \cap \operatorname{Ker}\left(\Phi_{\infty}\right)$ contains no lines; 
(ii) There is no sequence $\left(x_{n}\right)_{n \in \mathbb{N}} \in K$ such that

$$
\frac{x_{n}}{\left\|x_{n}\right\|} \rightarrow 0 \text { and } \frac{\kappa\left(\operatorname{dist}_{U}\left(x_{n}\right)\right)^{2}+\Phi\left(x_{n}\right)}{\left\|x_{n}\right\|} \rightarrow 0 ;
$$

and

(iii) $\langle f, u\rangle<\Phi_{\infty}(u), \quad \forall u \in\left(K_{\infty} \cap U\right), u \neq 0$.

From Theorem 3.2, it follows ([3], Corollary 5.1) that the stability of the existence of a solution is ensured if and only if the functional $\Psi-f$ is coercive.

This result relates the stability of the solution of a variational inequality and the coerciveness of an associated energy-type functional.

We conclude by noticing that the stable states for a semi-coercive inequality are precisely those which, due to the coercivity of the associated energy, are closer to the coercive case. It is our opinion that this link between stability and coercivity (already remarked in other contexts, see [1] for example) is not casual, being rather a very general feature.

\section{References}

1. Adly, S., Ernst, E. and Théra, M. (2001), A characterization of convex and semicoercive functionals, Journal of Convex Analysis, 8(1), 127-148.

2. Adly, S., Ernst, E. and Théra, M. (2000), Stabilité de l'ensemble des solutions d'une inéquations variationnelles non-coercive, Compte Rendu à l' Académie des Sciences Paris, 333, Série I 1, 409-414.

3. Adly, S., Ernst, E. and Théra, M. (2001), Stability of non-coercive variational inequalities, Communications in Contemporary Mathematics, 4(1), 145-160.

4. Adly, S., Goeleven, D. and Théra, M. (1996), Recession mappings and non-coercive variational inequality, Nonlinear Analysis T.M.A., 26, 1573-1603.

5. Auslender, A. and Crouzeix, J.-P. (1989), Well-behaved asymptotical convex functions, Ann. Inst. Henri Poincaré, 6, 101-121.

6. Brezis, H. (1968), Equations et inéquations non-linéaires dans les espaces vectoriels en dualité, Ann. Inst. Fourier, Grenoble, 18(1), 115-175.

7. Dieste1, J. (1975), Geometry of Banach Spaces, Selected Topics, Lecture Notes in Mathematics, Springer-Verlag, Berlin.

8. Zeidler, E. (1990), Nonlinear Functional Analysis and its Applications II, Springer-Verlag, Berlin.

9. Klee, V. (1955), Separation properties for convex cones, Proceedings of the American Mathematical Society, 6, 313-318.

10. Rockafellar, R.T. (1968), Convex Analysis, Princeton University Press. 